\title{
"Radiographic demonstration of association of ubernaculum Dentis (Gubernaculum tract) in odontogenic cysts and tumors"-A CBCT finding
}

\author{
Nagaraju Kamarthi, Dhruvesh Gupta1, Suhasini Palakshappa Gotur² \\ Departments of Oral Medicine and Radiology and ${ }^{2}$ Oral Pathology and Microbiology, Subharti Dental College, Swami Vivekanand \\ Subharti University, Meerut, Uttar Pradesh, 'Madhuban Dental And Orthodontic Centre, Delhi, India \\ Correspondence: Dr. Nagaraju Kamarthi, Department of Oral Medicine and Radiology, Subharti Dental College, Swami Vivekanand Subharti \\ University, Meerut, Uttar Pradesh, India. E-mail: Drnagrani1977@gmail.com
}

\section{ABSTRACT}

Spectrum of lesions that occur in the jaws have a cyst-like radiographic appearance. These lesions may be odontogenic or non-odontogenic and are often difficult to differentiate them on the basis of their clinical, radiographic features alone. Among odontogenic lesions without mineralization, ameloblastomas, odontogenic keratocysts, and dentigerous cysts can all appear as well-defined, unilocular, well-corticated, lucent lesions that can mimic with non-odontogenic cysts and tumors like nasopalatine duct cyst, aneurysmal bone cyst, central giant cell granuloma, hemangioma and so on. So understanding the pathogenesis of these lesions become the most imperative criteria for determining the additional investigations and treatment protocol. We hereby discuss 8 diagnosed cases of odontogenic and non-odontogenic jaw lesions, which were retrospectively visualized in cone beam computed tomography (CBCT), and an association of gubernaculum tract (cord) with odontogenic origin lesions was demonstrated.

Key words: Cone beam computed tomography; dental lamina; gubernaculum cord; gubernaculum dentis; gubernaculum tract

\section{Introduction}

Many lesions, both intraosseous and extraosseous, can involve the maxillary and mandibular regions of the jaw.Of these, odontogenic cysts and tumours are unique to the oral cavity and do not occur elsewhere in the body. These lesions originate from tissues involved in odontogenesis (i.e., tooth development) and include a wide variety of lesions ranging

\begin{tabular}{|c|c|}
\hline \multicolumn{2}{|c|}{ Access this article online } \\
\hline Quick Response Code: & \\
\hline & $\begin{array}{l}\text { Website: } \\
\text { www.ijri.org }\end{array}$ \\
\hline & $\begin{array}{l}\text { DOI: } \\
\text { 10.4103/ijri.IJRI_467_19 }\end{array}$ \\
\hline
\end{tabular}

from cystic lesions to hamartomas to non-neoplastic tissue proliferations and both benign and malignant neoplasms. ${ }^{[1,2]}$ Due to this wide range of biological behavior, there is currently much debate regarding the pathogenesis,

This is an open access journal, and articles are distributed under the terms of the Creative Commons Attribution-NonCommercial-ShareAlike 4.0 License, which allows others to remix, tweak, and build upon the work non-commercially, as long as appropriate credit is given and the new creations are licensed under the identical terms.

For reprints contact: WKHLRPMedknow_reprints@wolterskluwer.com

Cite this article as: Kamarthi N, Gupta D, Gotur SP. "Radiographic demonstration of association of ubernaculum Dentis (Gubernaculum tract) in odontogenic cysts and tumors"-A CBCT finding. Indian J Radiol Imaging 2020;30:340-3.

Received: 22-Nov-2019 Revised: 11-Feb-2020 Accepted: 13-May-2020 Published: 15-Oct-2020 
classification and clinical and histological variations of odontogenic cysts and tumours. ${ }^{[2]}$ Understanding of clinical, radiologic, and pathologic attributes of cysts and tumors of the jaws is comprehensive. A better understanding of the pathogenesis can have a significant effect in the treatment outcome.

\section{Gubernaculum dentis}

Significant histologic changes occur in the tissues overlying the erupting teeth. ${ }^{[3]}$ Initially, as the deciduous tooth erupts, the permanent tooth germ becomes situated apically and is enclosed by bone except for a small canal that is filled with connective tissue and often contains epithelial remnants of the dental lamina. This connective tissue mass, gubernaculum dentis, comprises of fibrous band (gubernacular cord) running in the bony channel (gubernacular canal or tract) that connects the pericoronal follicular tissue of the successional tooth with the overlying gingiva, and opens on the alveolar bone crest behind the deciduous predecessor. ${ }^{[4-6]}$ To summarize, gubernaculum dentis in the mixed dentition is an eruption pathway of the successional tooth.

\section{Radiographic demonstration of gubernaculum tract/canal}

The gubernacular tract (GT) is an eruption pathway from the dental follicle to the gingiva for permanent teeth. ${ }^{[6]}$ Various authors have described this normal structure in the textbooks, but very few focused on its presence, facade and significance. Alterations in the path, curve along with the obliteration of the canal has been demonstrated in the impacted and delayed erupting teeth. ${ }^{[7]}$ Radiologists must accurately identify these varied characteristics of GTs and aid in diagnosing so that accurate treatment protocol can be carried out.

In the last decade, 3-D (three-dimensional) imaging has aided in better visualization of bone structures, such as adjacent root positions, in order to locate canals and root fractures, as well as provide the ability to more accurately measure anatomical structures. A 3-D scan allows the clinicians to view the anatomy from different angles. Increasing availability of this technology provides the dental clinician with an imaging modality capable of providing a 3-dimensional representation of the complex anatomy of the maxilla and the mandible with minimal distortion. ${ }^{[8,9]}$

Cone beam computed tomography (CBCT) and multidetector computed tomography (MDCT) are considered some of the most reliable and useful 3-D imaging modalities for the examination of the maxilla and the mandible, including teeth - because of their ability to provide high-quality images of bone tissue and its adjacent structures. ${ }^{[8,9]}$ However, radiographic demonstration of role of GT in tooth eruption or its association with odontogenic lesions has not been elaborated by many authors.
Oda M, etal. (2018) verified the significance of imaging characteristics of GT and suggested that GT at the top of masses was useful not only for diagnosing odontogenic masses but also for differentiating odontogenic and non-odontogenic masses. ${ }^{[10]}$ They classified them into 3Groups viz. Group 1, Group 2 and Group 3[Figure 1] basing on the association of GT with lesions/masses. We followed the same criteria of grouping given by Oda etal. and visualized retrospectively the CBCT scans of 8 diagnosed cases (4 odontogenic and 4 non-odontogenic) of jaw lesions. Interestingly, CBCT scans revealed an association of gubernaculum tract with lesions of odontogenic origin and their position in the jaws did not affect its association.

Case 1 [Figure $2 \mathrm{~A}$ and B]: 2 cases of Odontomas, compound and complex in the mandibular anterior and posterior region showing an intact GT (Group 1).

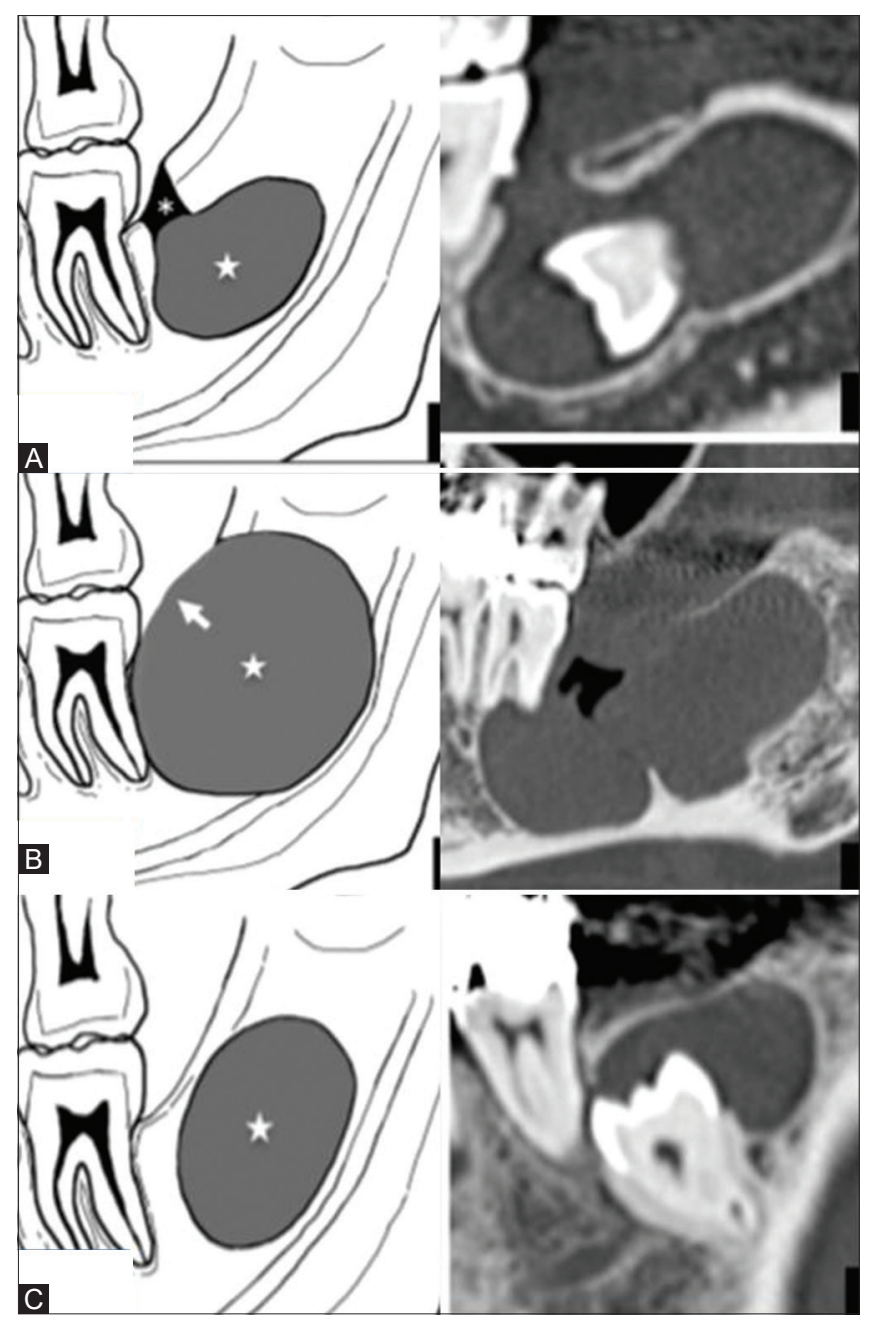

Figure 1 (A-C): Schematic and computed tomographic representation of association of GT with jaw lesions; Group 1 (A) Intact GT (asterisk) continues to the top of the mass (star); Group 2(B) Expanded GT (arrow) continues to the top of the mass (star); Group 3, (C) GT is not continuous with the tumor or cyst (star). (Courtesy of Oda M et al.) 
Case 2 [Figure 3]: 2 cases of unilocular radiolucent lesions involving with the mandibular third molar. Based on the clinical and radiographic features they were diagnosed as dentigerous cyst associated with impacted molar. Histopathological examination differentiated them as unicystic ameloblastoma. These lesions clearly demonstrated the presence of expanded GT association (Group 2).

Case3 [Figure 4] Two cases of dentigerous cyst; associated with mandibular third molar. One of the lesions has displaced the tooth into the ramus of the mandible (inferior to coronoid process) and the other involving the body and ramus of the mandible. Both the lesions clearly showed the expanded GT association (Group 2).

Case 4 [Figure 5A and B] A case of collateral odontogenic keratocyst in between lateral incisor and canine region. OPG could not demonstrate the continuity whereas CBCT scans could clearly demonstrate the presence of GT in these lesions.

Cases 5,6,7,8 [Figure 6A-D]:4 Cases of non-odontogenic origin, viz, central hemangioma, aneurysmal bone cyst (ABC), simple bone cyst (SBC), central giant cell granuloma (CGCG) respectively which have involved the jaws. Although the lesions were extensive involving the jaws, we could not demonstrate the association of GT with them.

\section{Discussion}

Etiopathogenesis of jaw lesions, either odontogenic or non-odontogenic, is a complex phenomenon and various
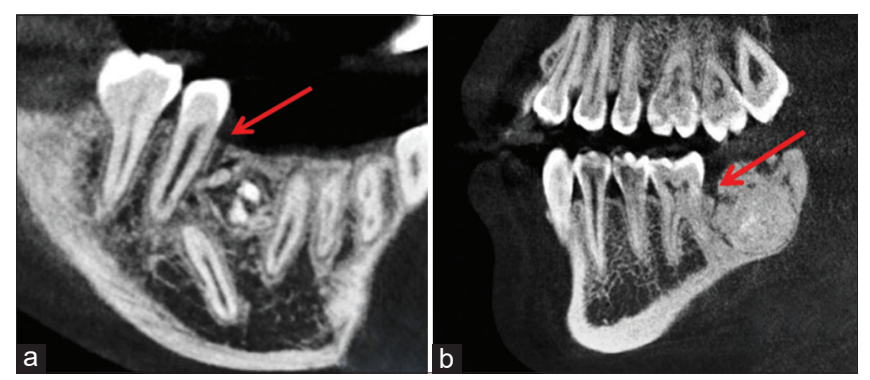

Figure 2 ( $A$ and $B$ ): GT association with odontomas; ( $A$ and $B$ ) CBCT demonstrating compound and complex odontomas with GT in the premolar molar region (red arrow)

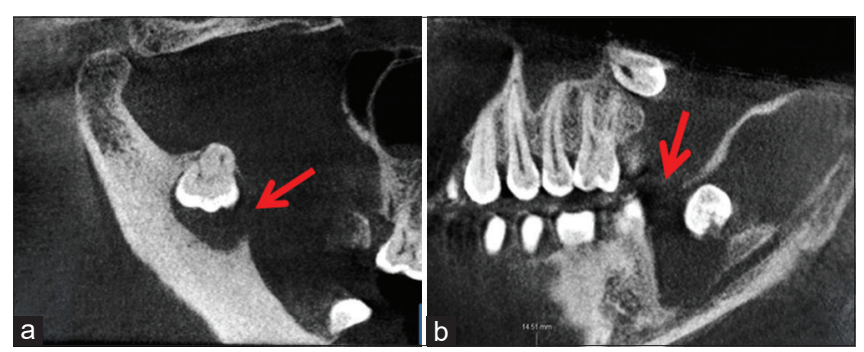

Figure 4 ( $A$ and $B$ ): CBCT scans of two cases of dentigerous cyst; in the ramus of the mandible $(A)$ and in the mandibular molar region $(B)$ theories and hypothesis have been put forward by the authors. A hypothetic role of Gubernaculum dentis (comprising chiefly of dental lamina) in the pathogenesis of ameloblastoma was suggested more than a century ago and in the recent past its association with odontogenic cysts (odontogenic keratocyst, dentigerous cyst) and odontogenic tumors (adenomatoid odontogenic tumor, odontomas) has been proposed. ${ }^{[11]}$ The significance of the image findings of the GT in relationship to odontogenic masses, including the possibility that the origin of the odontogenic mass might be the GT, was not taken into consideration as limited studies have been carried out on this facet. Limited resources and lack of advanced diagnostic aids can also be implicated in not arriving to the conclusion of this hypothesis.

With this concept, we retrospectively analyzed CBCT scans of (histologically diagnosed) 4 cases of odontogenic and 4 cases of non-odontogenic masses, to detect an association of GT.

Gubernaculum tract usually goes undetected in the 2-D images [Figure 5A] such as IOPAR (intra-oral periapical radiograph), OPG (Orthopantomograph).They can be better visualized on the advanced imaging modalities which can provide millimeter cut serial sectional images in three dimensional views. CBCT and MDCT scans can clearly demonstrate GT as acontiguous, corticated canal which is in continuous with the dental follicle of the unerupted teeth[Figures 2-5]. Owing to its limited radiation dose CBCT has revolutionized as an imaging modality of head and neck jaw lesions. ${ }^{[9]}$

So, we determined to visualize the spatial association of GT with odontogenic lesions by its imaging characteristics on
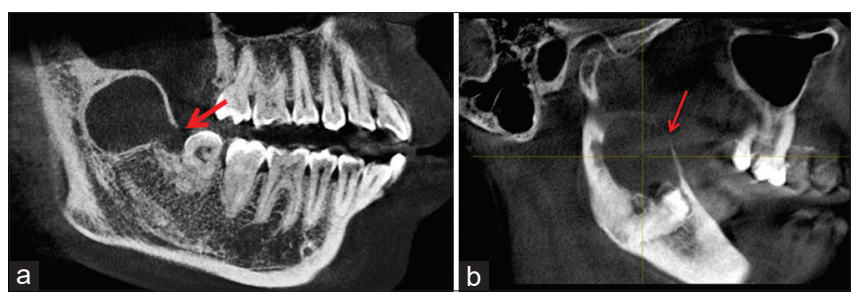

Figure 3 (A and B): CBCT images of Two cases of Unicystic Ameloblastoma with GT tract in association with mandibular third molar (red arrow)

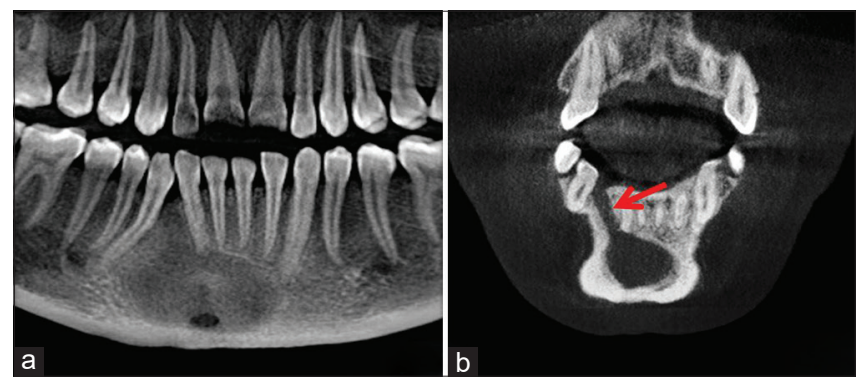

Figure 5 (A and B):(A and B) OPG and CBCT scan of Odontogenic keratocyst with clear demonstration of $\mathrm{GT}$ in CBCT scans 

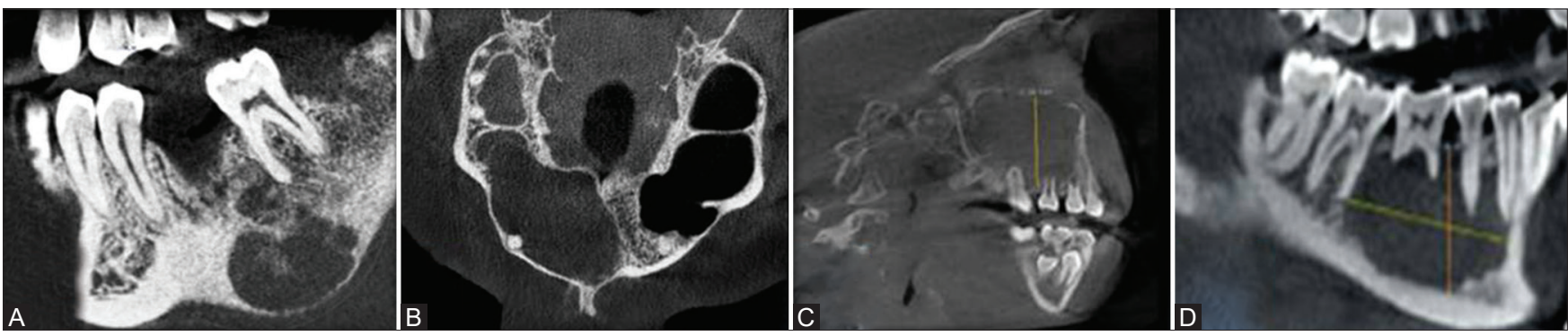

Figure 6 (A-D): CBCT scans (A-D) of Central hemangioma, ABC, SBC, CGCG respectively, with no GT association with lesions

CBCT. Histologically diagnosed cases of odontogenic and non-odontogenic cases where $\mathrm{CBCT}$ was performed were selected and were examined. Of the 4 cases of odontogenic origin we were able to clearly visualize an association of GT with these lesions in different sections either in axial, sagittal or coronal. However, association of GT was not evident in non-odontogenic masses [Figure 6A-D].

Role of finding an intact GT (Group 1) can clearly aid as a diagnostic criteria in confirming odontogenic origin whereas in Group 2, where expanded GT is associated with the masses, confusion can arise when the lesions are associated with massive cortical destruction. However, large non-odontogenic aggressive lesions [Figure 6A-D] demonstrated part of alveolar bone over the mass.

Previously, various authors have suggested that the lesions occurring above the inferior alveolar nerve canal and in the alveolar process region could possibly confirm odontogenic origin. ${ }^{[7]}$ In our cases, small odontogenic lesions either be in the alveolar process or in the ramus of the mandible [Figure 4] could clearly demonstrate the GT attached to the masses whereas large CGCG, ABC, TBC occurring in the alveolar process region could not demonstrate GT. These valid findings clearly signified in imparting the role of GT in odontogenic lesions.

\section{Conclusion}

Limitations of our finding were it was done in a retrospective manner and was confined to very limited cases and jaw lesions. Inference to impacted teeth, transposition of the teeth, and delayed eruption of teeth due to changes in the GT was not carried out. Modest consideration has been paid by physicians and radiologists regarding the image characteristics and spatial association of gubernacular tracts with odontogenic cysts and tumors. Based on our CBCT findings, we consider that, one should pay more attention to the association between the GT and odontogenic masses.
We expect that many more studies regarding this association will be performed in the future.

\section{Financial support and sponsorship}

Nil.

\section{Conflicts of interest}

There are no conflicts of interest.

\section{References}

1. Mosqueda-Taylor A. New findings and controversies in odontogenic tumors. Med Oral Patol Oral Cir Bucal 2008;13:E555-8.

2. El-Naggar AK, Chan JK, Grandis JR, Takata T, Slootweg PJ. WHO Classification of Head and Neck Tumours. $4^{\text {th }}$ ed. Lyon, France: IARC Press; 2017.

3. Broomell IN. Anatomy and Histology of the Mouth and Teeth. Philadelphia: Blakiston; 1898. p. 302-8.

4. Mummery JH. The Microscopic Anatomy of the Teeth. London: Oxford Univ Press; 1919. p. 23.

5. Scott JH, Symons NBB. Introduction to Dental Anatomy. $4^{\text {th }}$ ed. Edinburgh: Livingston; 1964. p. 97-9.

6. Nanci A. Ten Cate's Oral Histology. Development, Structure, and Function. $7^{\text {th }}$ ed. St. Louis: Mosby Elsevier; 2008. p. 79-289.

7. Oda M, Miyamoto I, Nishida I, Tanaka T, Kito S, Seta Y, et al. A spatial association between odontomas and the gubernaculum tracts. Oral Surg Oral Med Oral Pathol Oral Radiol 2016;121:91-5.

8. Junqueira RB, Verner FS, Campos CN, Devito KL, do Carmo AM. Detection of vertical root fractures in the presence of intracanal metallic post: A comparison between periapical radiography and cone-beam computed tomography. J Endodontics 2013;39:1620-4.

9. Brisco J, Fuller K, Lee N, Andrew D. Cone beam computed tomography for imaging orbital trauma-image quality and radiation dose compared with conventional multislice computed tomography. Br J Oral Maxillofac Surg 2014;52:76-80.

10. Oda M, Nishida I, Miyamoto I, Saeki K, Tanaka T, Kito S, et al. Significance and usefulness of imaging characteristics of gubernaculum tracts for the diagnosis of odontogenic tumors or cysts. PLoS One 2018;13:e199285.

11. White SC, Pharoah MJ. Oral Radiology-Principles and Interpretation. Chapter $15^{\text {th }}, 5^{\text {th }}$ ed.St. Louis: Mosby; 2004.p. 281-96. 